\title{
Three Dimensional Visualization of Bacterial Type III Export Apparatus in the Lyme Disease Spirochete Borrelia burgdorferi
}

\author{
Jiagang Tu ${ }^{1}$, Xiaowei Zhao ${ }^{1}$, Akarsh Manne ${ }^{2}$, Kathryn Lees ${ }^{2}$, Kai Zhang ${ }^{3}$, Chunhao Li $^{3}$, Steven J. Nortis ${ }^{1}$, \\ Md A. Motaleb ${ }^{2}$, Jun Liu ${ }^{1}$ \\ ${ }^{1}$ Department of Pathology and Laboratory Medicine, University of Texas Medical School at Houston, 6431 \\ Fannin Street, MSB 2.228, Houston, TX 77030. \\ ${ }^{2}$ Department of Microbiology and Immunology, Brody School of Medicine, East Carolina University, 600 Moye \\ Blvd., Greenville, NC 27834 \\ ${ }^{3}$ Department of Oral Biology, State University of New York at Buffalo, Buffalo, NY 14214
}

Type III secretion systems (T3SS) are widely used by Gram-negative bacteria to assemble flagella required for locomotion and to transport virulence effectors into eukaryotic cells [1]. The central component of these systems is the Type III secretion apparatus, which consists of a group of integral membrane proteins and three soluble proteins include a T3SS associated ATPase. Powered by proton motive force and ATP, the secretion apparatus plays essential role in substrates recognition and export. However, the structure and function of the intact secretion apparatus remain poorly understood at the molecular level.

In this study, the Lyme disease spirochete Borrelia burgdorferi was chosen as the model system [2] to study the molecular architecture of the export apparatus (Figure 1), because a) the T3SS genes are highly conserved among spirochetes and other bacterial species, b) its small cell diameter $(<0.3 \mu \mathrm{m})$ and multiple motors located at cell tips make it ideal for in situ structural analysis, and c) a novel genetic method is available to construct specific inframe deletion of any T3SS genes without the polar effect on downstream gene expression [3]. We constructed deletion mutants of every membrane protein in the Type III secretion apparatus of $B$. burgdorferi, and then determined their motor structures by using high-throughput cryo-electron tomography and sub-tomogram averaging [4].

Frozen-hydrated bacterial cultures were imaged at $-170^{\circ} \mathrm{C}$ using a $300 \mathrm{kV}$ electron microscope equipped with a field emission gun and a 16 megapixel CCD camera. Using the FEI "batch tomography" program, low-dose, single-axis tilt series were collected from each cell with a cumulative dose of $\sim 100 \mathrm{e}^{-} / \AA^{2}$. Tilt series were then aligned and reconstructed to generate 3-D cryo-tomograms by using a combination of IMOD [5] and RAPTOR [6]. In total, 223,015 images and $\sim 2700$ tomographic reconstructions were generated. One representative tomogram from $\triangle f l h A$ mutant is shown in Figure 2. More than 10,000 subvolumes of flagellar motors were extracted to determine three-dimensional structures of the flagellar motors in different mutants by sub-tomogram averaging [4].

Comparative structural analysis and molecular modeling reveal the first overall architecture of the secretion apparatus and the protein-protein interactions with unprecedented detail. This study represents a comprehensive approach toward understanding the fascinating and complex secretion apparatus in bacteria [7].

\section{References:}

[1] Erhardt, M., K. Namba and K. T. Hughes, Cold Spring Harb Perspect Biol (2000) 2(11): a000299.

[2] N. W. Charon, A. Cockburn, C. Li, J. Liu, K. A. Miller, M. R. Miller, M. A. Motaleb, C. W. Wolgemuth, Annu Rev Microbiol (2012) 66:349-70, 2012. 
[3] M. A. Motaleb, J. E. Pitzer, S. Z. Sultan and J. Liu, J Bacteriol (2011) 193(13): 3324-3331.

[4] X. Zhao, K. Zhang, T. Boquoi, B. Hu, M. A. Motaleb, K. Miller, M. James, N. W. Charon, M. D. Manson, S. J. Norris, C. Li, J. Liu, Proc Natl Acad Sci U S A, (2013) 110(35):14390-5.

[5] J. R. Kremer, D. N. Mastronarde and J. R. McIntosh, J Struct Biol (1996) 116(1): 71-76.

[6] F. Amat, F. Moussavi, L. R. Comolli, G. Elidan, K. H. Downing and M. Horowitz, J Struct Biol (2008) 161(3): 260-275.

[7] This work was supported by grants R01AI087946 from the NIAID and AU-1714 from the Welch Foundation.

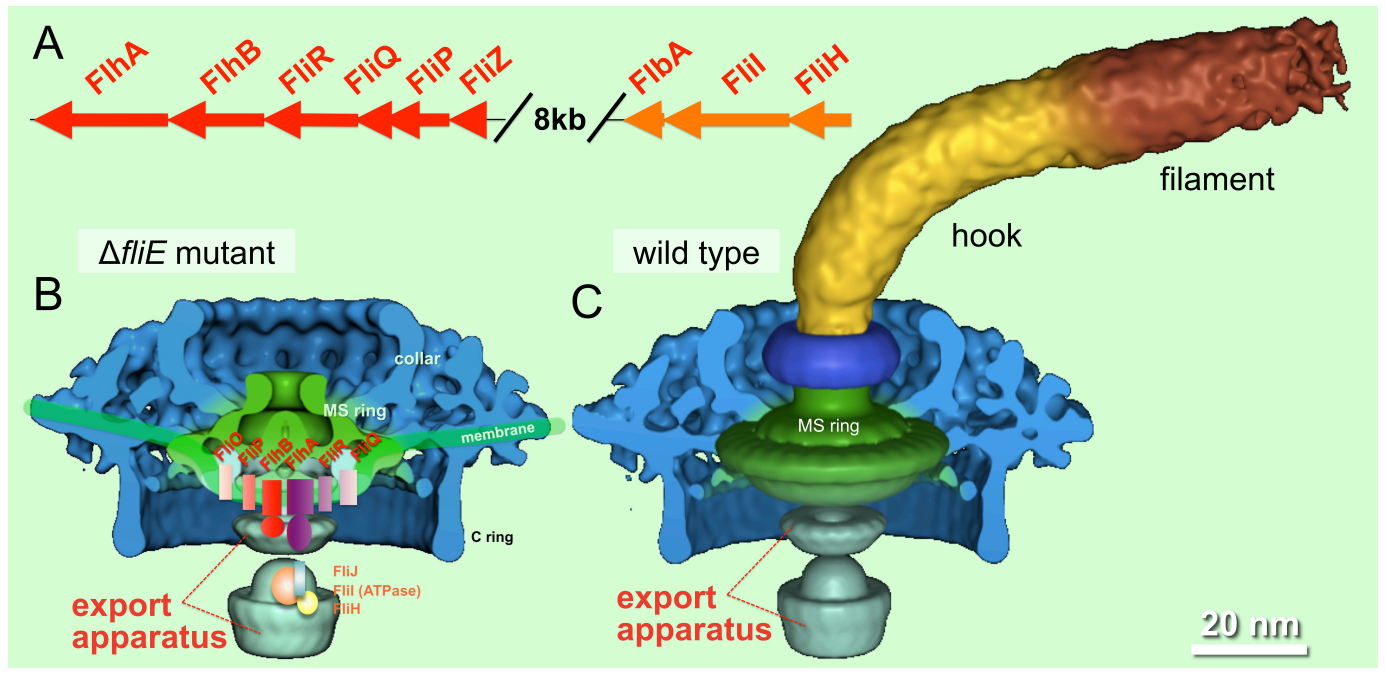

Figure 1. There are nine conserved T3SS genes in the genome of B. burgdorferi (A). Their gene products form the export apparatus, which is located at the base of the flagellar motors in $\Delta f l i E$ mutant (B) and wild type cells (C). The export apparatus is essential for the assembly of the hook and filament.

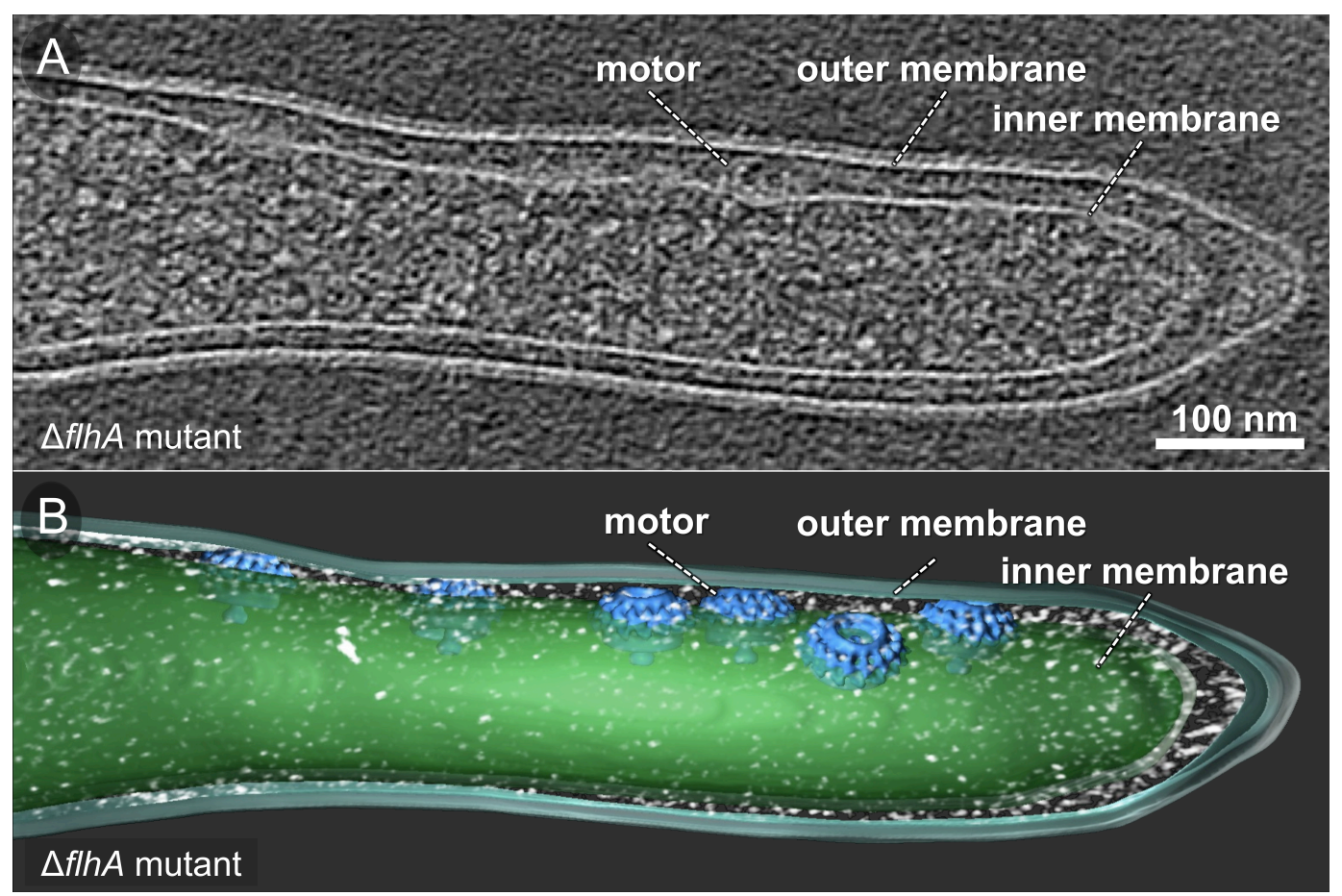

Figure 2. A 3D map from a $\triangle f l h A$ cell tip is shown in a central slice (A) and surface rendering (B). 